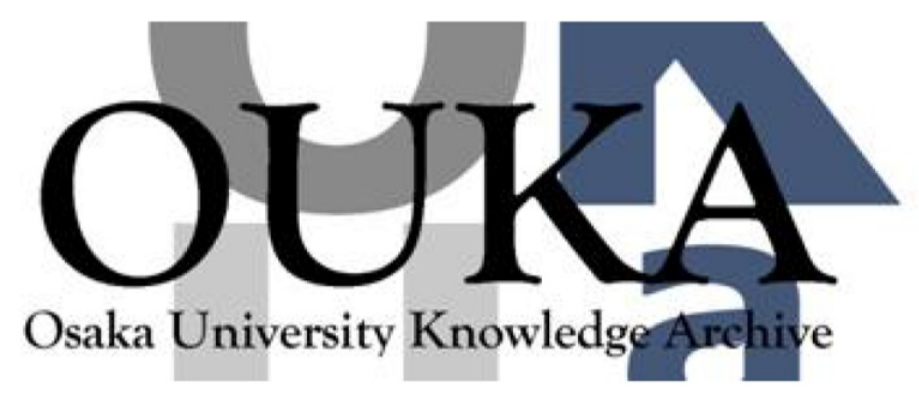

\begin{tabular}{|c|l|}
\hline Title & $\begin{array}{l}\text { Residual order in the thermal oxide of a fully } \\
\text { strained SiGe alloy on } \mathrm{Si}\end{array}$ \\
\hline Author(s) & $\begin{array}{l}\text { Shimura, Takayoshi; Okamoto, Yuki; Inoue, } \\
\text { Tomoyuki et al. }\end{array}$ \\
\hline Citation & Physical Review B. 81(3) p. 033308 \\
\hline Issue Date & $2010-01-15$ \\
\hline oaire:version & VoR \\
\hline URL & https://hdl. handle. net/11094/85489 \\
\hline rights & $\begin{array}{l}\text { Copyright } 2010 \text { by the American Physical } \\
\text { Society. }\end{array}$ \\
\hline Note & \\
\hline
\end{tabular}

Osaka University Knowledge Archive : OUKA

https://ir. Library. osaka-u. ac. jp/

Osaka University 


\title{
Residual order in the thermal oxide of a fully strained SiGe alloy on $\mathrm{Si}$
}

\author{
Takayoshi Shimura, * Yuki Okamoto, Tomoyuki Inoue, Takuji Hosoi, and Heiji Watanabe \\ Department of Material \& Life Science, Osaka University, 2-1 Yamadaoka, Suita, Osaka 565-0871, Japan \\ (Received 15 December 2009; revised manuscript received 30 December 2009; published 27 January 2010) \\ Residual order in the thermal oxide of a fully strained SiGe alloy on a $\mathrm{Si}(001)$ surface was investigated by \\ synchrotron x-ray diffraction. Ordered $\mathrm{SiO}_{2}$ was present in the oxide layer, and the crystalline order was \\ similar to that of Si. The dependence of the order on oxidation time and temperature was also obtained. On the \\ basis of these results, the oxidation reaction at the interface and the differences in rate enhancement between \\ dry and wet oxidation conditions are discussed.
}

DOI: 10.1103/PhysRevB.81.033308

PACS number(s): 81.65.Mq, 61.05.cm, 68.35.Fx, 68.55.aj

\section{INTRODUCTION}

The oxidation of SiGe alloys has been investigated for both fundamental and technological reasons, as has Si oxidation. It has been expected as the fabrication process for the gate oxide of SiGe channel metal-oxide field-effect transistors (MOSFETs), which have higher carrier mobility than $\mathrm{Si}$ channel MOSFETs. ${ }^{1}$ In recent years, it has also been employed in the fabrication of SiGe-on-insulator and Ge-oninsulator substrates., ${ }^{2,3}$ These substrates have enabled highspeed MOSFETs with channel materials of strained $\mathrm{Si}, \mathrm{SiGe}$, and Ge. ${ }^{4}$

However, the oxidation mechanism of SiGe alloy is still open to question, ${ }^{5}$ and clarification of the mechanism is more difficult than with Si due to the added complexity induced by lattice strain and diffusion of Ge atoms. The primary characteristic of $\mathrm{SiGe}$ oxidation is the ejection of $\mathrm{Ge}$ atoms from the interface between the surface oxide and the SiGe layer, without their incorporation into the oxide layer. Ge atoms accumulate at the $\mathrm{SiO}_{2} / \mathrm{SiGe}$ interface, and diffuse into the $\mathrm{SiGe}$ layer. ${ }^{1,2}$ In strained $\mathrm{SiGe}$ layers epitaxially grown on $\mathrm{Si}$ substrates, the effects of strain relaxation and defect generation during oxidation must be taken into account.

The oxidation rate of SiGe alloy is significantly higher than that of Si under wet oxidation conditions, but is almost equal or slightly higher under dry oxidation. ${ }^{1,5-7}$ This enhancement of wet oxidation increases with increasing initial Ge concentration in the alloy. This could be interpreted as an effect of the Si-Ge bond weakness, compared to the Si-Si bond. However, bond weakness does not explain the difference between wet and dry oxidation, because it should result in enhancement of the rate under both conditions. A point defect suppression effect on SiGe oxidation has also been proposed, ${ }^{1}$ but this was not widely accepted.

It is important to investigate the atomic structure of the thermally oxidized layer, because doing so allows us to understand what occurs at the oxidized interface. In a thermally oxidized $\mathrm{Si}$ layer, the $\mathrm{Si}$ atoms in the oxide layer maintain their order, which originates from the diamond structure of the parent $\mathrm{Si}$ crystal, although the structure appears to be amorphous at first glance. ${ }^{8,9}$ This provides a clearer picture of the oxidation process and explains the nearly perfect properties obtained by electric measurements of the $\mathrm{SiO}_{2} / \mathrm{Si}$ interface.

In the present study, the residual order in the thermal oxide layer on a fully strained SiGe alloy was investigated by synchrotron x-ray diffraction. The dependence of this order on the oxidation time and temperature is demonstrated. We also obtained information regarding the strain relaxation process of the SiGe layer and the diffusion of Ge atoms. On the basis of these results, the oxidation reaction at the interface and the mechanism of SiGe oxidation rate enhancement are discussed.

\section{EXPERIMENT}

$\mathrm{Si}_{1-x} \mathrm{Ge}_{x} \quad(x=0.13)$ layers were epitaxially grown on $\mathrm{Si}(001)$ substrates by chemical vapor deposition. The $\mathrm{SiGe}$ layers were $85 \mathrm{~nm}$ thick, and fully strained, so that the lateral lattice spacing of the SiGe layers was exactly the same as that of the $\mathrm{Si}$ substrate. These samples were oxidized in dry $\mathrm{O}_{2}$ at temperatures from 850 to $1000{ }^{\circ} \mathrm{C}$.

Synchrotron $\mathrm{x}$-ray diffraction experiments were performed on beam line $4 \mathrm{C}$ of the Photon Factory, KEK, Tsukuba, Japan, employing a four-circle diffractometer with an $\mathrm{Si}(111)$ crystal analyzer. The wavelength was chosen to be $0.154 \mathrm{~nm}$. The intensity distributions along the crystal truncation rod (CTR) scattering elongated from the 111 Bragg point were measured under the symmetric condition, in which the incident and outgoing angles were equal. Diffraction peaks from the ordered $\mathrm{SiO}_{2}$ and the fully strained $\mathrm{SiGe}$ layer were observed in the CTR scattering. Therefore, we obtained information regarding the crystalline order of the $\mathrm{SiO}_{2}$ and $\mathrm{SiGe}$ layers simultaneously.

We also obtained reciprocal space maps around the 113 Bragg reflections to evaluate the crystalline order of the SiGe layers. The measurements were carried out by using a fourcircle diffractometer installed on a $18-\mathrm{kW}$ rotating anode $\mathrm{x}$-ray generator. $\mathrm{X}$ rays of $\mathrm{Cu} \mathrm{K} \alpha$ radiation were selected by a multilayered mirror and a $\mathrm{Ge}(220)$ channel-cut monochromator. A Ge(220) channel-cut analyzer was also employed in front of the detector. The maps were obtained under the asymmetric condition of small incidence angle.

\section{RESULTS AND DISCUSSION}

Figure 1 shows the intensity distributions along the CTR scatterings elongated from the 111 Bragg points for the $\mathrm{Si}_{1-x} \mathrm{Ge}_{x} / \mathrm{Si}(x=0.13)$ and $\mathrm{Si}$ substrates. Figure 1(b) shows an enlargement around the 111 Bragg point. The diffraction peaks of the SiGe layer were near those of the Si substrate, 

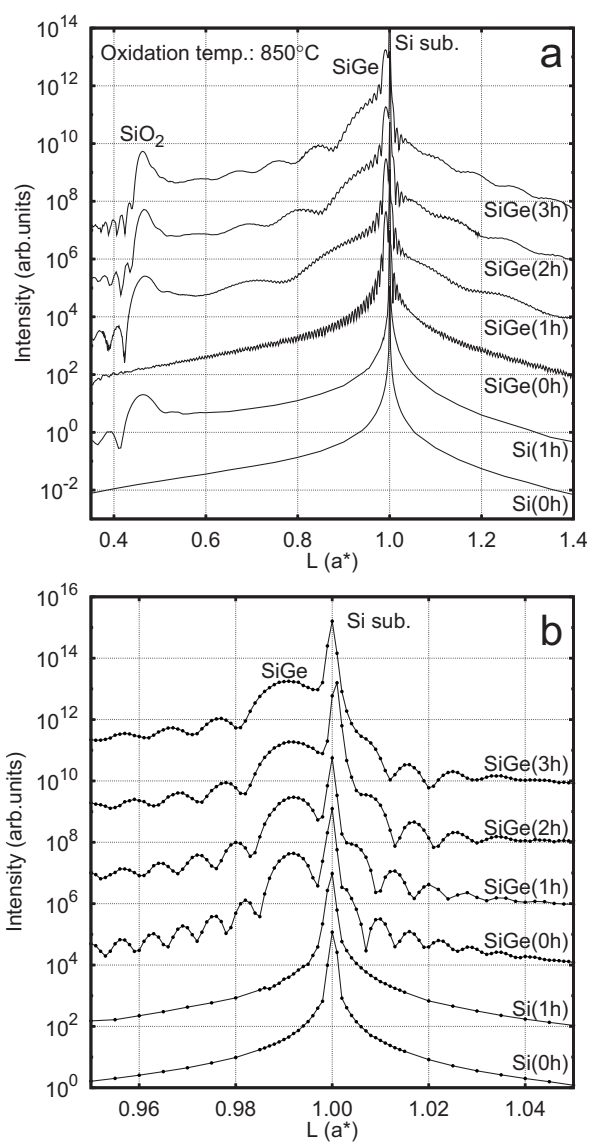

FIG. 1. (a) Intensity distributions of CTR scatterings elongated from the 111 Bragg point of $\mathrm{Si}_{1-x} \mathrm{Ge}_{x} / \mathrm{Si}(x=0.13)$ and $\mathrm{Si}$ substrates oxidized at $850{ }^{\circ} \mathrm{C}$ for 0 to $3 \mathrm{~h}$. (b) Enlargement around the 111 Bragg point of (a).

and their position did not change during oxidation, whereas the period of the intensity oscillation became larger. This indicates that the $\mathrm{SiGe}$ layer became thinner as oxidation progressed, but it maintained the original lattice strain.

Another intensity oscillation was recognized in the distribution from the oxidized $\mathrm{SiGe} / \mathrm{Si}$ sample, for which the period was approximately $\Delta L=0.18$ after $1 \mathrm{~h}$ of oxidation [see Fig. 1(a)]. The period of this oscillation became smaller with increasing oxidation time, and the peak centers of the patterns were located around the 111 Bragg point of the substrate. Therefore, this oscillation was a thickness fringe in the diffraction pattern from the interfacial $\mathrm{SiGe}$ layer, with higher $\mathrm{Ge}$ fraction, located at the $\mathrm{SiO}_{2} / \mathrm{SiGe}$ interface. The thickness of this layer was estimated from the period of the oscillation, and the Ge fraction was obtained by spectroscopic ellipsometer. The thickness gradually increased with increasing oxidation time, and the Ge fraction was approximately $40 \%$, as shown in Fig. 2.

The thicknesses of the $\mathrm{SiO}_{2}$ and $\mathrm{SiGe}$ layers were also estimated from the periods of their oscillation patterns. Table I shows the relative amounts of $\mathrm{Si}$ and $\mathrm{Ge}$ atoms during the oxidation, obtained based on the thickness, Ge fraction, and strain of the samples. Since the oxide surface usually lacked crystalline order, the oxide layer was actually slightly thicker than our estimation. ${ }^{10}$ It is possible that some Ge atoms

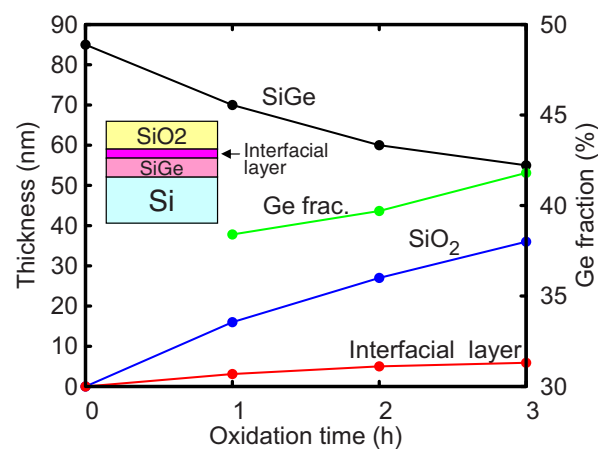

FIG. 2. (Color online) Thickness of surface oxide, interfacial layer, SiGe layers, and Ge fraction of the interfacial layer during oxidation.

evaporated from the SiGe surface during the initial stage of thermal oxidation. The determined values are reasonable to within the accuracy of the measurements, and demonstrate the validity of the estimations.

Diffraction peaks showing the residual order in the oxide layers were clearly observed around $L=0.45$ for the oxidized $\mathrm{SiGe}$ samples. The intensity and the position of the peaks for the sample oxidized for $1 \mathrm{~h}$ were nearly identical to those of the $\mathrm{SiO}_{2} / \mathrm{Si}$ sample. This indicates that the residual order in the oxide layers of both samples had the same crystalline order. The intensity of the diffraction peak gradually increased and the period of oscillation decreased with increasing oxidation time. This indicates that ordered $\mathrm{SiO}_{2}$ was formed at the interface for further oxidation.

The residual order in the thermal oxide layer of the $\mathrm{Si}$ substrates originates from the diamond structure of the parent Si crystal. During the Si oxidation process, oxygen atoms are inserted between the $\mathrm{Si}$ atoms and the bond configuration is relaxed to release the resulting strain. However, the fundamental Si-O network configuration is maintained, indicating that the structural rearrangement caused by the bond breaking and rebonding is not dominant. In our previous studies we showed that the residual order in the Si oxide layer is an unstable transition state. ${ }^{9,11}$ Thermal annealing above $950{ }^{\circ} \mathrm{C}$ for $1 \mathrm{~h}$ causes degradation of the residual order, transforming it into an amorphous structure. We also reported degradation by diffusion of atomic oxygen through the oxide layer. ${ }^{12}$ These results indicate that the ordered $\mathrm{SiO}_{2}$ did not crystallize from the amorphous structure during the oxidation process. Instead, the ordered $\mathrm{SiO}_{2}$ was in the transition state from the diamond $\mathrm{Si}$ structure to the amorphous oxide.

In $\mathrm{SiGe}$ oxidation, the $\mathrm{SiGe}$ substrate has the same atomic configuration as the diamond structure of $\mathrm{Si}$. However, the Ge atoms are ejected from the oxide interface into the substrate. Therefore, limited ordering is expected in the SiGe

TABLE I. Relative amounts of Si and Ge atoms in the samples during oxidation.

\begin{tabular}{ccccc}
\hline \hline Oxidation time (h) & 0 & 1 & 2 & 3 \\
\hline $\mathrm{Si}$ & 1 & 0.93 & 0.89 & 0.89 \\
$\mathrm{Ge}$ & 1 & 0.92 & 0.93 & 0.98 \\
\hline \hline
\end{tabular}




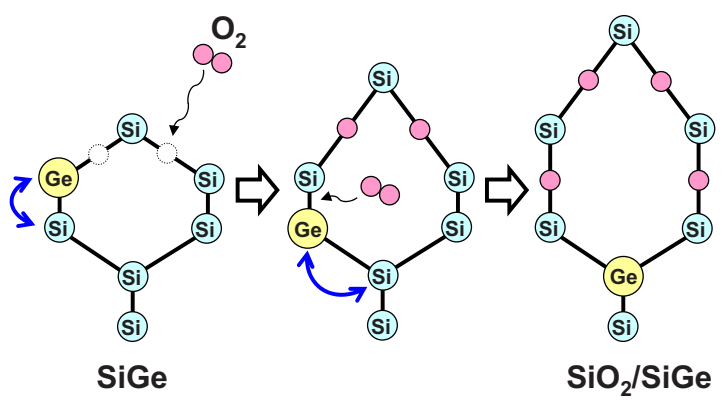

FIG. 3. (Color online) Schematic drawing of diffusion of Ge atoms at the interface into the substrate during oxidation.

oxide layer. However, the crystalline order is the same as that of the Si oxide layer, as shown in Fig. 1. This indicates that the Ge atoms at the oxide interface switched positions with adjacent $\mathrm{Si}$ atoms before the oxygen atoms were inserted into the back bond of Ge atoms (Fig. 3). The replacement of Ge atoms incorporated in the oxide by $\mathrm{Si}$ atoms would degrade the order, because the ordered $\mathrm{SiO}_{2}$ was in the transition state. The Ge fraction at the interface after $2 \mathrm{~h}$ oxidation was approximately $40 \%$, while the ordered $\mathrm{SiO}_{2}$ formed continuously. This indicates that the replacement of $\mathrm{Ge}$ atoms by $\mathrm{Si}$ atoms below the interface occurred, even when the Ge fraction was $40 \%$.

Figure 4 shows the dependence of the crystalline order of $\mathrm{SiGe}$ and $\mathrm{SiO}_{2}$ layers on the oxidation temperature. The diffraction peak of the SiGe layer became broad, and the oscillation pattern disappeared after oxidation at $1000{ }^{\circ} \mathrm{C}$. The angular width perpendicular to the CTR scattering also broadened (Fig. 5). These results indicate that the SiGe layer degraded during high-temperature oxidation, which enhanced the diffusion of atoms and the strain relaxation.

Correspondingly, the diffraction peak of the ordered $\mathrm{SiO}_{2}$ broadened, and the oscillation pattern disappeared after hightemperature oxidation. This degradation was not due to thermal effects, but was mainly caused by degradation of the SiGe layer, because the diffraction peak of the ordered $\mathrm{SiO}_{2}$ on the Si substrate oxidized at $950{ }^{\circ} \mathrm{C}$ had a clear oscillation pattern in our previous studies. ${ }^{11}$ The crystalline order of the $\mathrm{SiO}_{2}$ depends on that of the $\mathrm{SiGe}$ layer, since it maintains the residual order of the parent crystal. However, it should be noted that the residual order remains in the oxide layer, even after high-temperature oxidation.

Recently, a new linear-parabolic rate equation for the thermal oxidation of $\mathrm{Si}$ in dry $\mathrm{O}_{2}$ was proposed by Watanabe et $a l .{ }^{13}$ In the Deal-Grove model, ${ }^{14}$ the oxidation rate is limited by the interfacial oxidation reaction at the initial stage of oxidation. Therefore, the oxide thickness increases linearly with time. At longer oxidation times, the rate is limited by the diffusion of $\mathrm{O}_{2}$ through the thick oxide layer. In the model proposed by Watanabe et al., the oxidation rate is not limited by the oxidation reaction, but by the diffusion of $\mathrm{O}_{2}$ through a strained oxide region near the $\mathrm{SiO}_{2} / \mathrm{Si}$ interface over a short oxidation time. No rate-limiting step due to interfacial reaction was included in their model based on firstprinciples calculations; the activation barrier for the interfacial oxidation reaction is negligibly small. ${ }^{15,16}$ The interface reaction by oxidizing species of $\mathrm{H}_{2} \mathrm{O}$ molecules limits the
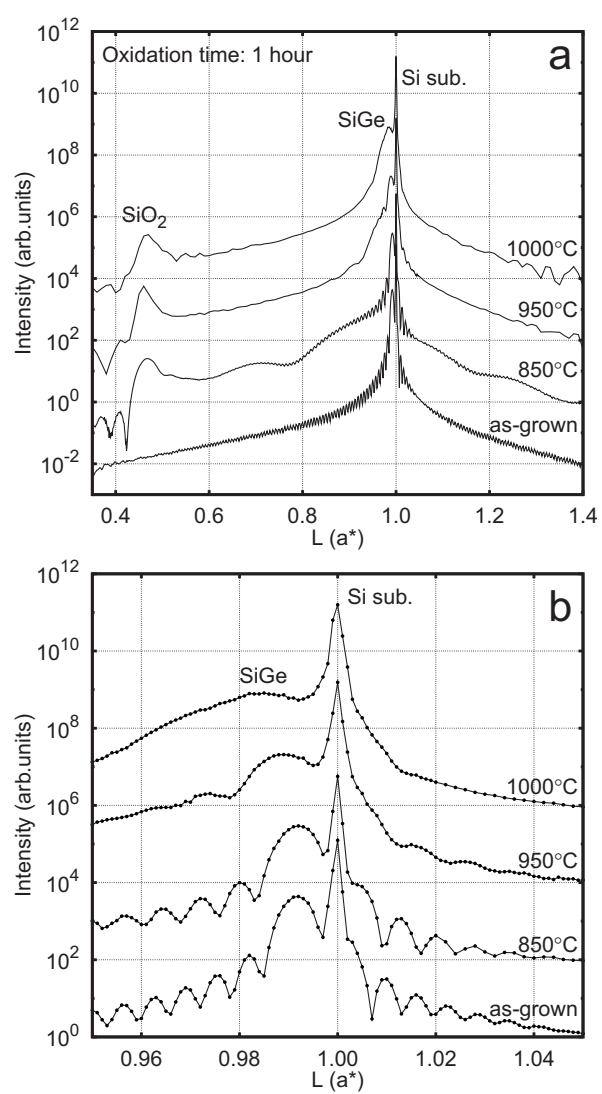

FIG. 4. (a) Intensity distributions of CTR scatterings elongated from the $111 \mathrm{Bragg}$ point of $\mathrm{Si}_{1-x} \mathrm{Ge}_{x} / \mathrm{Si}(x=0.13)$ and $\mathrm{Si}$ substrates oxidized for $1 \mathrm{~h}$ at 850 to $1000{ }^{\circ} \mathrm{C}$. (b) Enlargement around the 111 Bragg point of (a).

wet oxidation rate, according to the Deal-Grove model.

Based on this model, the difference in the rate enhancement between dry and wet oxidation can be easily explained. The results of the present study showed that the atomic structure of the thermal oxide on SiGe alloy and on Si substrates were nearly identical. This indicates similar oxidation rates, because of equivalent diffusivity of $\mathrm{O}_{2}$ through strained $\mathrm{SiO}_{2}$ near the interface. However, for SiGe alloy, the enhancement of the oxidation rate in wet oxidation is due to the weak bonding between $\mathrm{Si}$ and $\mathrm{Ge}$.

There is an inconsistency with respect to the dry oxidation rate of SiGe alloy in the previous reports. LeGoues et al. showed that the oxidation rate of SiGe alloy was the same as that of $\mathrm{Si}^{6}$ while other reports showed a slight enhancement of the SiGe oxidation rate. ${ }^{17}$ We speculate that this inconsistency appeared because the crystalline order of the SiGe layer was not monitored, and this order depends upon the epitaxial growth conditions, oxidation temperature, and $\mathrm{Ge}$ fraction. The oxidation rate thus depends upon the crystalline order of the SiGe layer, even if the oxidation conditions are exactly the same. This is because the rate is limited by diffusion through the interfacial $\mathrm{SiO}_{2}$, which depends upon the crystalline order of the SiGe layer. In our results the oxidation rate of the $\mathrm{SiGe}$ layer was slightly higher than that of $\mathrm{Si}$ during the oxidation at $850{ }^{\circ} \mathrm{C}$ for $1 \mathrm{~h}$. We considered that this was due to slight degradation of the $\mathrm{SiGe}$ layer. Figure 6 shows the reciprocal space maps around the 113 reflections 

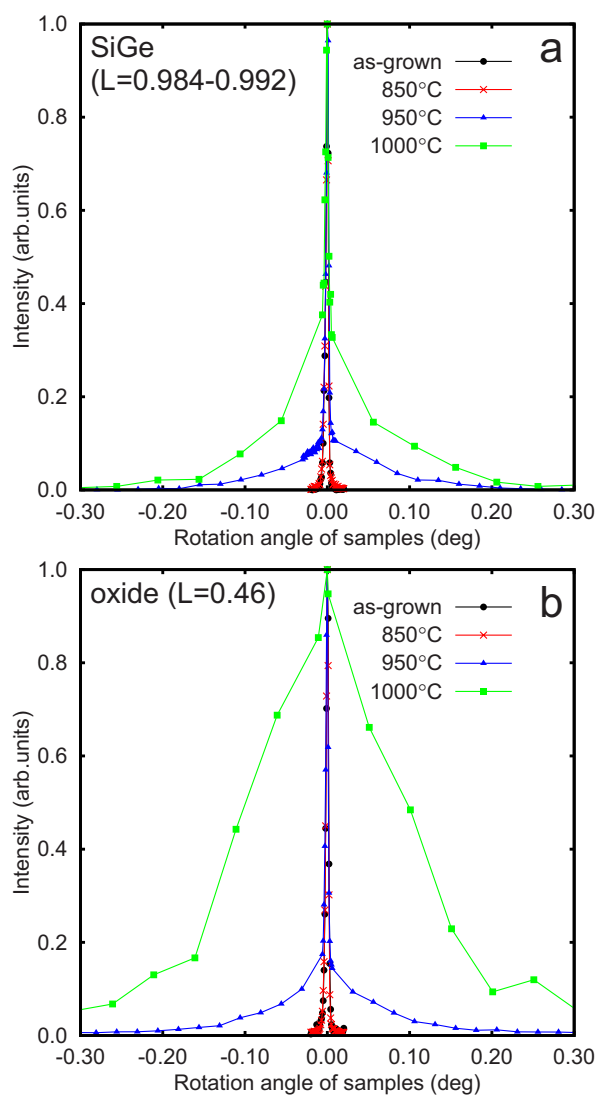

FIG. 5. (Color online) Intensity profiles perpendicular to the CTR scatterings of the $\mathrm{Si}_{1-x} \mathrm{Ge}_{x} / \mathrm{Si}(x=0.13)$ samples. (a) and (b) show the profiles at the $\mathrm{SiGe}$ and the ordered $\mathrm{SiO}_{2}$ peaks, respectively. The intensities are normalized at the peak positions.

of the as-grown sample and the sample oxidized at $850{ }^{\circ} \mathrm{C}$ for $1 \mathrm{~h}$. We can see the broad and very weak tail around the SiGe Bragg reflection of the oxidized sample, indicating the slight degradation of the SiGe layer.

In conclusion, the diffraction peak from the residual order in the thermal oxide of $\mathrm{SiGe} / \mathrm{Si}$ was observed by synchrotron $\mathrm{x}$-ray diffraction. The residual order had the same crystalline order as the $\mathrm{Si}$. This indicates that the $\mathrm{Ge}$ atoms at the oxide interface switched positions with the nearest $\mathrm{Si}$ atoms before the oxygen atoms were inserted into the back bond of $\mathrm{Ge}$ atoms. The difference in the rate enhancement between dry
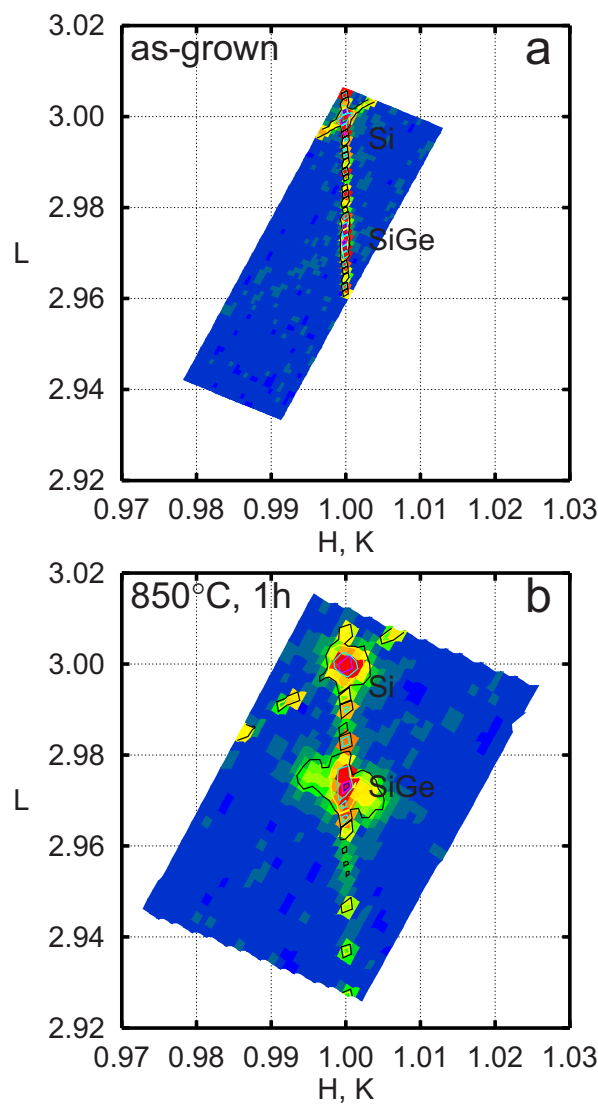

FIG. 6. (Color online) Reciprocal space maps around the 113 Bragg reflections. (a) and (b) show the maps of the as-grown sample and the sample oxidized at $850{ }^{\circ} \mathrm{C}$ for $1 \mathrm{~h}$, respectively. The contour levels are $0.5,1.0,5.0,50.0,100.0$, and 1000.0 counts/sec.

and wet oxidation can be explained based on Watanabe's rate-limiting model.

\section{ACKNOWLEDGMENTS}

This work was supported partly by the Global Center of Excellence (COE) Research Projects, Center for Atomically Controlled Fabrication Technology, of MEXT of Japan. The synchrotron radiation experiments were performed at the Photon Factory (Proposals No. 2006G057 and No. 2008G017).

\footnotetext{
*shimura@mls.eng.osaka-u.ac.jp

${ }^{1}$ F. K. LeGoues et al., J. Appl. Phys. 65, 1724 (1989).

${ }^{2}$ T. Tezuka et al., Jpn. J. Appl. Phys. 40, 2866 (2001).

${ }^{3}$ S. Nakaharai et al., Appl. Phys. Lett. 83, 3516 (2003).

${ }^{4}$ M. L. Lee et al., J. Appl. Phys. 97, 011101 (2005).

${ }^{5}$ E. Napolitani et al., J. Appl. Phys. 97, 036106 (2005).

${ }^{6}$ F. K. LeGoues et al., Appl. Phys. Lett. 54, 644 (1989).

${ }^{7}$ D. K. Nayak et al., Appl. Phys. Lett. 57, 369 (1990).

${ }^{8}$ K. Tatsumura et al., Phys. Rev. B 69, 085212 (2004).

${ }^{9}$ T. Shimura et al., ECS Trans. 1, 39 (2005).
}

\footnotetext{
${ }^{10}$ I. Takahashi et al., J. Phys.: Condens. Matter 5, 6525 (1993).

${ }^{11} \mathrm{~K}$. Tatsumura et al., Jpn. J. Appl. Phys. 42, 7250 (2003).

${ }^{12}$ K. Tatsumura et al., Phys. Rev. B 72, 045205 (2005).

${ }^{13}$ T. Watanabe et al., Phys. Rev. Lett. 96, 196102 (2006).

${ }^{14}$ B. E. Deal and A. S. Grove, J. Appl. Phys. 36, 3770 (1965).

${ }^{15}$ A. Bongiorno and A. Pasquarello, Phys. Rev. Lett. 93, 086102 (2004).

${ }^{16}$ T. Akiyama and H. Kageshima, Surf. Sci. 576, L65 (2005).

${ }^{17}$ M. Spadafora et al., Mater. Sci. Semicond. Process. 8, 219 (2005).
} 